\title{
Establishment of an in-house, recombinant nucleocapsid protein-based enzyme linked immunosorbent assay (ELISA) for the detection of chicken infectious bronchitis
}

\author{
G. Haqshenas \\ Virology section, Department of Bacteriology and Virology, National Research Center for Genetic Engineering and \\ Biotechnology (NRCGEB), Tehran, Iran.
}

Accepted 8 March, 2013

\begin{abstract}
Infectious bronchitis (IB) is one of the devastating chicken viral diseases that causes significant financial loses to chicken industry worldwide. In this study, highly purified recombinant nucleocapsid protein (rNP) of IB virus (IBV) was used to develop an in-house enzyme linked immunosorbent assay (iELISA) for the detection of IB. The iELISA was optimized to achieve maximum sensitivity and specificity. All tested broiler chicken farms were positive for IBV antibody in a commercial ELISA kit (cELISA) and the optimized iELISA. Thirty three (33) specific pathogen free (SPF) chickens and 20 broiler chickens held in a restricted environment tested negative for IBV antibody in both ELISA. The above results suggest that the iELISA is a sensitive and specific test for the detection of antibody to IBV in chicken flocks.
\end{abstract}

Key words: Enzyme linked immunosorbent assay (ELISA), infectious bronchitis (IB), recombinant nucleocapsid protein (rNP).

\section{INTRODUCTION}

Infectious bronchitis (IB), a highly contagious upper respiratory disease, was first reported in 1931 (Schalk and Hawn, 1931; Collisson et al., 1992; Cavanagh and Naqi, 2003) and continues to be one the major threats to chicken industry worldwide. The disease has been described in young and laying chickens with age-dependent clinical signs. In young chickens, common respiratory signs including tracheal rales, sneezing and coughing are observed. The virus may also affect non-respiratory organs including kidneys and oviducts of young chickens (Darbyshire et al., 1979). IBV-induced clinical signs in adult laying chickens are poor egg quality and significant reduction in egg production (Ignjatovic and Sapats, 2000; Hofstad, 1984).
IBV (genus coronavirus, family Coronaviridae) (Cavanagh and Naqi, 2003) contains $27.6 \mathrm{~kb}$ single stranded positive sense RNA (Boursnell et al., 1987). Many serotypes have been reported for IBV (Cavanagh, 2007; Ignjatovic and Sapats, 2000). The genome encodes three major structural proteins; the 150 to $200 \mathrm{kDa}$ spike (S) glycoprotein, the 20 to $30 \mathrm{kDa}$ integral membrane glycoprotein (M), and the 43 to $50 \mathrm{kDa}$ nucleocapsid (N) phosphoprotein (Cavanagh, 1981; Spaan et al., 1988; Saif, 1993). The immunodominant N protein (NP) induces cross-reactive antibodies (Ignjatovic and Galli, 1994) and enhances the protection level when it is expressed endogenously (Tang et al., 2008; Yang et al., 2009). The recombinant full length NP of IBV gray strain 
has been used to develop an enzyme linked immunosorbant assay, ELISA (Ndifuna et al., 1998). Also, partial NP has been utilized for the development of an IBV ELISA (Lugovskaya et al., 2006).

IBV infects chicken flocks worldwide. The disease has been reported from chicken flocks in Iran using conventional serological and virological methods (Aghakhan et al., 1994; Momayez et al., 2002; Vasfi and Bozorgmehri, 2000). Using molecular techniques, we and other research groups have detected and differentiated Iranian IBV isolates (Ghahremani et al., 2011). Despite the extensive use of IBV live attenuated and inactivated vaccines to protect commercial chicken flocks in Iran, IB remains responsible for serious financial losses to the poultry industry of the country. This study tested whether the full length recombinant NP (rNP) derived from the H120 strain of IBV can be used to develop an ELISA for the detection of IBV antibody.

\section{MATERIALS AND METHODS}

\section{Recombinant IBV N protein}

Expression of the recombinant IBV NP (rNP-IBV) was performed using a bacterial expression system as described previously (Haqshenas et al., 2004). Briefly, the full length $\mathrm{N}$ gene of IBV strain H120 was reverse transcription-polymerase chain reaction (RT$\mathrm{PCR}$ ) amplified and cloned into the $\mathrm{pET}-23 \mathrm{a}(+)$ plasmid. The final construct was named pET-23a-NP. To prepare the recombinant $\mathrm{N}$ protein for ELISA, Escherichia coli BL21(D3) bacteria were transfected with the pET-23a-NP construct and grown using Lauryl broth (LB) medium supplied with $50 \mu \mathrm{g} / \mathrm{ml}$ of ampicillin. The expression of protein was induced using isopropyl- $\beta$ - $D$-thiogalactopyranoside (IPTG )at $30^{\circ} \mathrm{C}$ overnight. The rNP contained a six-histidine tag at its carboxyl terminus (C-terminus) allowing the purity of the protein by affinity chromatography using $\mathrm{Ni}+$ resin as previously described ( Haqshenas et al., 2004). Following elution of the protein in the elution buffer ( $50 \mathrm{mM} \mathrm{NaH} \mathrm{PO}_{4}, 300 \mathrm{mM} \mathrm{NaCl}$; $\mathrm{pH}$ 8.0) containing $250 \mathrm{mM}$ imidazole, the protein was quantified using the Bradford method (Bradford, 1976). The aliquots of the purified protein were stored at $-70^{\circ} \mathrm{C}$ and the required amounts were thawed for the performance of the optimization experiment and the detection of IBV antibody.

\section{Serum samples}

Two hundreds and twenty seven (227) serum samples were received from 21 broiler chicken farms by Iranian Veterinary Organization (IVO), Tehran. Thirty three (33) serum samples were collected from specific pathogen free (SPF) chickens reared in specific isolators located at the central laboratory of the IVO. Twenty samples were collected from 15-day-old broiler chickens held in a restricted environment. All above samples were tested for IBV antibody using the well established whole virus-based IDEXX IBV commercial ELISA kit (cELISA) (IDEXX, Westbrook, ME) by IVO according to the manufacturer's instructions.

\section{Optimization of iELISA}

To achieve the maximum sensitivity and specificity for iELISA, several factors were optimized. The amount of antigen per each well is critical for the optimal detection of antibody. To determine the optimal quantities of antigen, the rNP purified by affinity chromatography (Haqshenas et al., 2004) was diluted in carbonate buffer $(\mathrm{pH}$ 9.6) to final concentrations $0.25,0.5,1,2,4$ and $8 \mu \mathrm{g} / 100 \mu \mathrm{l}$ buffer. $100 \mu \mathrm{l}$ of the above antigen preparations were added to each well of 96-well plate (Nunc). The plates were incubated at $37^{\circ} \mathrm{C}$ for $2 \mathrm{~h}$. The unbound antigen was removed by one wash with PBS. The unfilled areas were blocked using blocking reagent $(5 \%$ skimmed milk, $4 \%$ rabbit serum in PBS, $\mathrm{pH} 7.4$ ) overnight at $4{ }^{\circ} \mathrm{C}$. The coated wells were washed twice with PBS-T $(0.05 \%$ Tween-20 in PBS, $\mathrm{pH} 7.4$ ) and twice with PBS. $100 \mu \mathrm{I}$ IBV-positive primary antibody (Haqshenas et al., 2013) diluted in $0.5 \mathrm{x}$ blocking reagent $(1 / 400)$ were added to each well. Following $30 \mathrm{~min}$ at $37^{\circ} \mathrm{C}$, wells were washed as above. Horse-radish peroxidase (HRP)-conjugated, rabbit anti-chicken secondary antibody (Sigma) was diluted using $0.5 \mathrm{x}$ blocking reagent. $100 \mu \mathrm{l}$ of the diluted secondary antibody were added to each well and incubated for $30 \mathrm{~min}$ at $37^{\circ} \mathrm{C}$. The wells were washed as above. The developing solution containing 2,2'-azinodi(3-ethyl benzthiazoline sulfonic acid) (ABTS) chromogen (Sigma) in citrate buffer $(\mathrm{pH} 4.2)$ and $0.1 \% \mathrm{H}_{2} \mathrm{O}_{2}$ were added according to the manufacturer's instructions. The plates were incubated for $15 \mathrm{~min}$ at room temperature before the absorbance values were measured at $405 \mathrm{~nm}$ using an ELISA reader. To determine the optimal concentration of primary antibody, serial twofold dilutions $(1 / 50$ to $1 / 102400)$ of the positive serum (Haqshenas et al., 2004) were tested. One microgram antigen was used to coat each well and ELISA was performed as described above. To determine the optimal concentration of secondary antibody, it was serially two-fold diluted (1/250-1/512,000). ELISA was formed as above using $1 \mu \mathrm{g}$ antigen per well and primary antibody was diluted $1 / 400$. Throughout this study, a control negative excluding primary antibody was included as the background control and the obtained OD value was subtracted from the positive and negative OD values.

\section{Examination of clinical serum samples for IBV antibody}

Serum samples received from IVO were diluted in $5 \mathrm{x}$ blocking reagent to a final concentration of $1 / 400$ and tested in duplicate. Using $1 \mu \mathrm{g}$ antigen per well and secondary antibody at dilution $1 / 500$, iELISA was performed as described above. A conservative cutoff value of 0.16 (mean of OD values of negative samples, 0.07 , plus six standard deviations, 0.015) was set as described previously (Haung et al.,2002). The OD values below and above the cutoff were considered negative and positive, respectively.

\section{RESULTS AND DISCUSSION}

\section{Optimization of iELISA}

Optimization of ELISA for the achievement of maximum specificity and sensitivity using minimum quantities of reagents is a critical step in the establishment of a new ELISA. To optimize iELISA, optimal concentrations of several reagents including antigen, primary and seconddary antibodies were determined. We aimed to produce a signal not exceeding $1 \mathrm{OD}$. To identify the best concentration of antigen per well, the highly purified rNP was prepared using affinity chromatography as described (Haqshenas et al., 2004). SDS-PAGE and immunoblot analysis revealed that in consistent with our previous report (Haqshenas et al., 2004) the antigen preparation was highly pure (data not shown). A wide range of antigen quantities ( 0.25 to $8 \mu \mathrm{g}$ per well) were utilized for the determination of the best antigen concentration per well. 
A

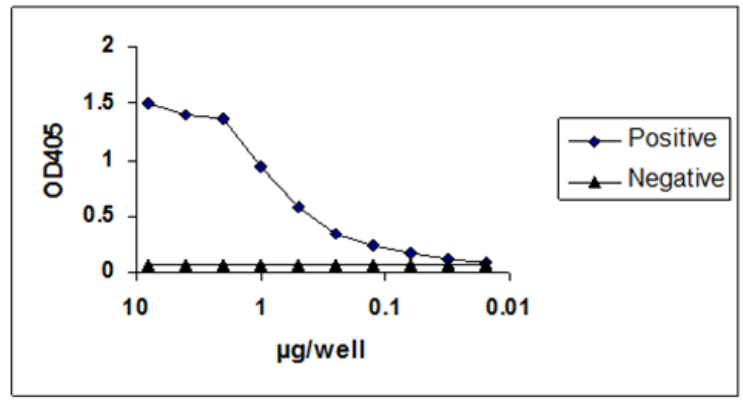

B

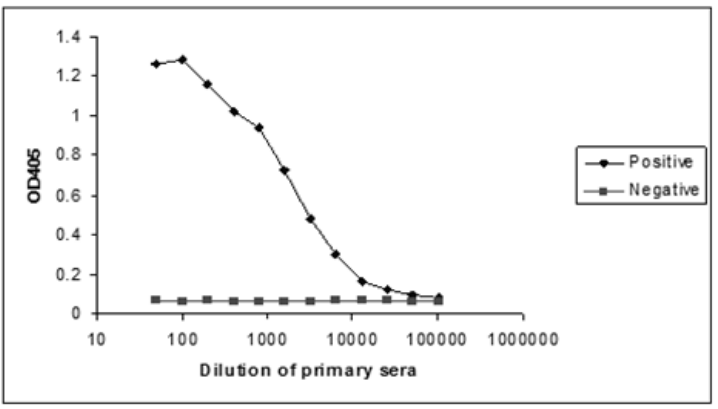

C

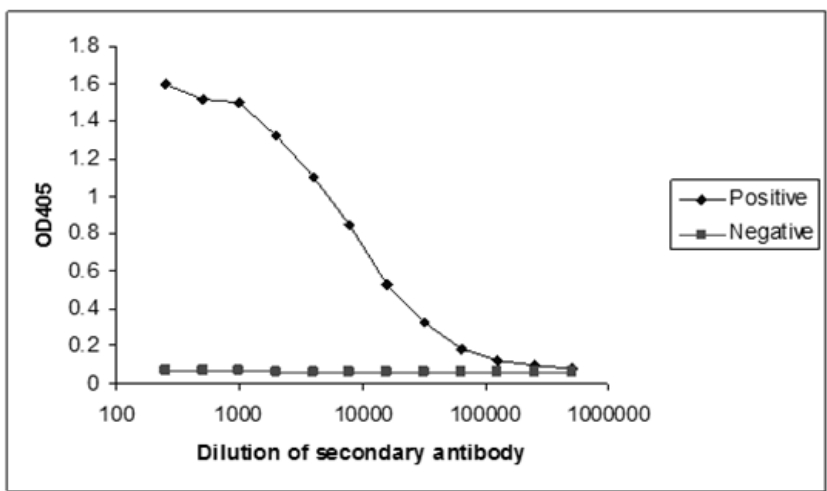

Figure 1. Optimization of in-house ELISA. (A) Various concentrations of the recombinant IBV N protein (rNP-IBV) were used for the optimization of antigen concentration. (B) The IBV-positive serum sample (Haqshenas et al., 2013) was serially two-fold diluted and tested using $1 \mu \mathrm{g}$ antigen per well and 1/500 secondary hP-conjugated antibody. (C) Using $1 \mu \mathrm{g}$ antigen per well and primary antibody at dilution 1/400, serially two-fold diluted secondary antibody was tested. iELISA was performed as described in Materials and Method.

As can be seen from Figure 1, $1 \mu \mathrm{g}$ antigen per well produced $\sim 1$ OD (Figure 1A) and represented the optimal antigen concentration for the performance of iELISA. The background $O D$ value using negative serum sample remained the same for all antigen quantities (Figure 1A). Subsequently, the best primary and secondary antibody dilutions were determined to be $1 / 400$ (Figure 1B) and $1 / 5000$ (Figure 1C), respectively. The above dilutions produced approximately 1 OD value and the background signal did not exceed ones obtained using other dilutions.

\section{Detection of antibody to IBV in clinical serum samples}

ELISA is the most economical and convenient test for the examination of large numbers of clinical serum samples for the presence of antibody. Several cELISA kits are available from different companies. However, the commercial kits are expensive and some circumstances may disrupt their export and import. We have successfully developed an ELISA using bacterially expressed avian hepatitis $E$ virus (HEV) ORF2 protein for the detection of avian HEV antibody (Haung et al., 2002). Recently, we developed a highly sensitive and specific rNP-IBV based dot-blot assay for the detection of antibody to chicken IBV in clinical serum samples (Haqshenas et al., 2013). The current study was designed to use the IBV rNP to deveIop an iELISA kit for the serological detection of IB disease and the immune response status to the IBV vaccination. The NP was used in this assay because it is highly conserved among IBV strains with 94 to $99 \%$ identity (Williams et al., 1992; Sneed et al., 1989). Therefore, the rNP based iELISA can be potentially used to serologically identify chickens infected with any IBV strain although further experimental data are required to confirm this point. Following opitimization, to evaluate the sensitivity and specificity of iELISA, 227 clinical serum samples from 21 chicken farms were examined for IBV antibody using the cELISA and iELISA. All 21 tested chicken farms (227 serum samples) were found to possess antibody to IBV antibody in both ELISA suggesting $100 \%$ sensitivity and specificity of the iELISA in the detection of IBV-antibody positive chicken flocks. The above results suggest a high correlation between the two ELISAs (100\% agreement). In addition, the results of iELISA and CELISA on the individual 227 serum samples were compared. Using the cELISA, 204 and 23 serum samples tested positive and negative for the presence of IBV antibody, respectively. In the iELISA, 209 and 18 sera were positive and negative, respectively. Seven out 
Table 1. 227 serum samples from 21 chicken farms were tested by the commercial ELISA (cELISA) and in-house ELISA (iELISA).

\begin{tabular}{ccccc}
\hline & \multicolumn{4}{c}{ iELISA } \\
\cline { 2 - 5 } & & Pos & Neg & Total \\
\hline \multirow{3}{*}{ cELISA } & Pos & 202 & 2 & 204 \\
& Neg & 7 & 16 & 23 \\
& Total & 209 & 18 & 227 \\
\hline
\end{tabular}

Pos, Positive; Neg, negative.

of the 23 samples negative in the cELISA produced OD values above the cutoff in the iELISA. These seven samples were also positive for IBV antibody when they were tested by two additional tests dot-blot and sodium dodecyl sulfate polyacrylamide gel electrophoresis (SDSPAGE)/immunoblot analyses (Haqshenas et al., 2013). SDS-PAGE/immunoblot analysis revealed that these sera specifically interacted with the IBV NP (Haqshenas et al., 2013). Therefore, including these seven samples, the apparent sensitivity of iELISA was higher than the cELISA (209 vs 204 out of 227 samples) and approximately $2 \%$ of the positive samples remained undetected in the cELISA. The failure of the cELISA to detect these seven samples might be due to the difference in the coating antigen that has been used by the kits; the cELISA uses the whole IBV while iELISA uses the rNP. These seven chickens might have serocoverted to the $\mathrm{N}$ protein more efficiently than to the outer proteins like the $S$ glycoprotein. Consequently, these seven samples were detected in the iELISA but not in the cELISA. Of 204 positive samples in the CELISA, 202 samples produced OD values above the cutoff in the iELISA. This difference could be due to technical error or lower specificity of cELISA. Also, there is a possibility that these two chickens seroconverted to the other viral structural proteins more efficiently than to the $\mathrm{N}$ protein. Unfortunately, due to lack of access to the antigen used by the IDEXX Laboratories, it was impossible to investigate the specific interaction of antibody and IBV antigen. A summary of the results has been presented in Table 1 . To test the specificity of the iELISA, 33 serum samples from SPF chickens and 20 serum samples collected from chickens held in a restricted environment were tested by both ELISA. All the tested samples were negative for IBV antibody using both ELISA. The above results suggest $100 \%$ specificity for the iELISA. Additionally, all IBVnegative serum samples containing high antibody titers to Newcastle disease virus (NDV), infectious bursal disease virus (IBDV), and influenza virus produced OD values below the cutoff further indicating high specificity of iELISA. In conclusion, the above results demonstrate that iELISA is a specific and sensitive test for the detection of IBV antibody and can be widely used as a substitution for the commercial kits.

\section{ACKNOWLEDGEMENTS}

This study was support by an internal grant from National Research Centre for Genetic Engineering and Biotechnology. We wish to thank the IVO for providing chicken serum samples.

\section{REFERENCES}

Aghakhan SM, Afshar Fereidouni NA, Marunesi C, Khodashenas M (1994). Studies on avian viral infections in Iran. Archives de L, Institute Razi 44/45: 1-10

Boursnell MEG, Browns TDK, Foulds IJ, Green PE, Tomley FM, Binns MM (1987). Completion of the sequence of the genome of the coronavirus avian infectious bronchitis virus. J. Gen. Virol. 68:57-77

Bradford MM (1976). A rapid and sensitive method for the quantification of microgram quantities of protein. Anal. Biochem. 72:248

Cavanagh D (1981). Structural polypeptides of coronavirus IBV. J. Gen. Virol. 53:93-103

Cavanagh, D (2007). Coronavirus avian infectious bronchitis virus. Vet. Res. 38: 281-297

Cavanagh, D and Naqi, SA (2003). Infectious bronchitis, in: Saif Y.M., Barnes H.J., Glisson J.R., Fadly A.M., McDougald L.R., Swayne D.E. (Eds.), Diseases of poultry, lowa, $11^{\text {th }}$ edition, Ames, lowa State University Press, 2003. pp. 101-119

Collisson E, Williams A, Parr R, Wang L (1992). An overview of the molecular biology of infectious bronchitis virus. Poult. Sci. Rev. 4: 41-55

Darbyshire JH, Rowell JG, Cook JKA, Peters RW (1979). Toxonomic studies on strains of avian infectious bronchitis virus using neutralizations tests in tracheal organ cultures. Arch. Virol. 61:227238

Ghahremani N, Bozorgmehri Fard MH, Shoushtari $H$, Momayez R, Sheiki N, Khoshzahmat A, Eshratabadi F (2011). Molecular analysis of infectious bronchitis virus isolated in Iran from 1998-2008. J. Anim. Vet. Adv. 10: 2961-67

Haqshenas G, Akrami H, Shayegh M (2004). Molecular Cloning and Expression of Nucleocapsid Gene of Chicken Infectious Bronchitis Virus Strain Massachusetts H120. J. Sci. I.R. Iran. 15:211-218. ISSN 1016-1104

Haqshenas G, Akrami H, Hedayati M, Farshian A (2013). Development of a Recombinant Protein-based Dot-blot Hybridization Assay for the Detection of Antibody to Chicken Infectious Bronchitis Virus. Iranian J. Vet. Res. (In Press)

Hofstad MS (1984). Cross-immunity in chickens using seven isolates of avian infectious bronchitis virus. Avian Dis. 25:650-654

Ignjatovic J, Galli L (1994). The S1 glycoprotein but not the N or M proteins of avian infectious bronchitis virus induces protection in vaccinated chickens. Arch. Virol. 138:117-34

Ignjatovic J, Sapats S (2000). Avian infectious bronchitis virus. Rev. Sci. Tech. 19:493-508

Lugovskaya NN, Scherbakov AV, Yakovleva AS, Tsyvanyuk MA, Mudrak NS, Drygin VV, Borisov AV (2006) Detection of antibodies to avian infectious bronchitis virus by a recombinant nucleocapsid protein-based enzyme-linked immunosorbent assay. J. Virol. Methods 135:292-296

Martin NL, Rautonen J, Crombleholme W, Rautonen N, Wara DW (1992). A screening test for the detection of anti-HIV-1 IgA in young infants. Immunol. Invest. 21:65-70

Momayez, R; Pourbakhsh, SA ; Kodashenas, and R ; Banani, M (2002). Isolation and identification of infectious bronchitis isolates from commercial flocks. Archives of Razi Institute 53: 1-9.

Ndifuna A, Waters AK, Zhou M, Collisson EW (1998). Recombinant nucleocapsid protein is potentially an inexpensive, effective serodiagnostic reagent for infectious bronchitis virus. J. Virol. Methods 70:37-44.

Saif LJ (1993). Coronavirus immunogens. Vet. Microbiol. 37:285-97.

Schalk AF, Hawn MC (1931). An apparently new respiratory disease of baby chicks. J. Am. Vet. Med. Assoc. 78:413-422 
Sneed LW, Butcher GD, Parr R, Wang L, Collisson EW (1989). Comparisons of the structural proteins of avian infectious bronchitis virus as determined by western blot analysis. Viral Immunol. 2:221227.

Spaan W, Cavanagh D, Horzinek MC (1988). Coronaviruses: structure and genome expression J. Gen. Virol. 59:2939-2952

Tang M, Wang H, Zhou S, Tian G (2008). Enhancement of the immunogenicity of an infectious bronchitis virus DNA vaccine by a bicistronic plasmid encoding nucleocapsid protein and interleukin-2. J Virol Methods 149:42-8.

Vasfi Marandi M, Bozorgmehri Fard M (2000). Isolation and identification of infectious bronchitis viruses in chickens in Iran. Proceeding of the World's poultry congress 2000. Montreal, Canda. August 20-25
Williams AK, Wang L, Sneed LW, Collisson EW (1992). Comparative analyses of the nucleocapsid genes of several strains of infectious bronchitis virus and other coronaviruses. Virus Res. 15:213-22.

Yang T, Wang HN, Wang X, Tang JN, Gao R, Li J, Guo ZC, Li YL (2009). Multivalent DNA vaccine enhanced protection efficacy against infectious bronchitis virus in chickens. J. Vet. Med. Sci. 71:1585-90. 